\title{
Evaluation of the efficacy of recombinant tissue plasminogen activators in improving clinical status of acute ischemic stroke: $A$ randomized clinical trial in Iran
}

\author{
Mojtaba Khazaei $^{1}$, Zaher Khazaei ${ }^{2}{ }^{*(}{ }^{(}$, Elham Goodarzi $^{3}$, Ali Ghadimi ${ }^{4}$
}

\section{${ }^{1}$ Assistant Professor of neurology Hamadan University of Medical Sciences, Hamadan, Iran \\ ${ }^{2} \mathrm{MSc}$ in Epidemiology, Student Research Committee, Sabzevar University of Medical Sciences, Sabzevar, Iran \\ ${ }^{3}$ Department of Health and Community Medicine, Faculty of Medicine, Dezful University of Medical Sciences, Dezful, Iran \\ ${ }^{4}$ Medical Student, Hamadan University of Medical Sciences, Hamadan, Iran \\ Correspondence}

Zaher Khazaei, MSc in Epidemiology, Student Research Committee, Sabzevar University of Medical Sciences, Sabzevar, Iran

Email: zaherkhazaei@yahoo.com

\section{History}

- Received: 05 May 2018

- Accepted: 20 August 2018

- Published: 29 September 2018

DOI :

https://doi.org/10.15419/bmrat.v5i9.481

\section{Check for updates}

\section{Copyright}

(c) Biomedpress. This is an openaccess article distributed under the terms of the Creative Commons Attribution 4.0 International license.

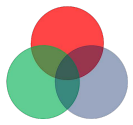

BioMedPress

The Open Access Publisher

\begin{abstract}
Background: Acute ischemic stroke is caused by blockage of a cerebral artery and is also known as cerebrovascular accident (CVA). Recombinant tissue plasminogen activator ( $r$-PA) therapy is effective in reducing early and long-term neurologic disabilities if it is started quickly. Therefore, the aim of this study was to determine the efficacy of treatment with recombinant tissue plasminogen activators in improving the clinical status of acute ischemic stroke. Methods: The current study was performed as a clinical trial of two groups- treatment and control ( $n=20$ per group). The treatment group consisted of patients who received rt-PA, while the control group consisted of patients who did not receive rt-PA. For each group, evaluation of neurological disabilities following ischemic stroke was based off the National Institutes of Health Stroke Scale (NIHSS), for early assessment of disability on the third day of treatment), and off the modified Rankin Scale (MRS) at 90 days after stroke. The drug effect criterion was used to reduce the neurological disability or the difference in NIHSS on day 3 after treatment. Also, the duration of onset of symptoms until the arrival of the patients to the emergency room (ER), as well as the risk factors, complications and number of deaths in both groups, were recorded. Data obtained were analyzed by SPSS software. Results: The results of the study showed that the mean of ER arrival time, NIHSS before treatment, and NIHSS on day 3 of treatment in the control group was higher than that of the treatment group; the difference was statistically significant $(P<0.05)$. The results also indicated that the probability of improvement of neurological disabilities in the experimental group was greater than that of the control group (relative risk $(R R)=1.25)$. Additionally, the odds ratio $(O R)$ for receiving rt-PA in the NIHSS positive treatment group compared to the control group was equal to $1 / 5(\mathrm{OR}=1.5)$. The results showed that $30 \%$ of the patients in the treatment group were treated with a complication. The mean of MRS was higher in the control group at 90 days after the stroke, compared with the treatment group. Conclusion: Treatment with rt-PA reduces the neurological disability in patients with ischemic stroke, since the mean of MRS is lower in the treatment group, compared with the control group, after 90 days of treatment.
\end{abstract}

Key words: Acute ischemic stroke, Iran, Neurologic disability, Randomized clinical trial, Recombinant tissue plasminogen activator

\section{INTRODUCTION}

Stroke is a medical emergency caused by blockade or reduction of blood flow to the brain tissue. Stroke is the second leading cause of death worldwide, with around 55 million deaths each year, of which about $10 \%$ are due to stroke ${ }^{1}$. The disease has a vascular origin and lasts more than 24 hours. It is a major cause of physical disability throughout the world and is the main cause of long-term hospitalizations, which can significantly increase the cost of treatment ${ }^{2}$. Risk factors associated with the disease include increased age, gender, high blood pressure, diabetes mellitus (DM), smoking, obesity, low triglycerides, high cholesterol diet, high salt diet, alcohol consumption, family history, atrial fibrillation, and oral contraceptive pills
$(\mathrm{OCP})^{3-5}$. The most common type of stroke is ischemic stroke, which consists of $85 \%$ of all strokes and is divided into two types (thrombotic and embolic) ${ }^{6}$. Since 1950, with the introduction of effective therapies for blood pressure, there has been a significant reduction in the incidence of stroke. However, with the increase in age, the death rate from stroke is expected to double by 2030 .

In the United States, nearly 7800,000 cases of stroke occurred annually, of which $87 \%$ are ischemic and only $13 \%$ are hemorrhagic. Also, it leads to 150000 deaths in the United States. It is estimated that one of each 16 deaths which occurs in the United States, is due to stroke. While few studies in Iran have been conducted in this field, it has been reported that the 
prevalence of stroke in Iran is 43 cases per 100,000 people $^{7-9}$.

Indeed, stroke is one of the most common causes of mortality and disability worldwide; more than $80 \%$ of all patients are diagnosed with ischemic stroke ${ }^{9,10}$. Considering the high mortality and morbidity of cerebral stroke, treatments for this disease are crucial. Therapies are targeted at regenerating blood flow, and can significantly improve the mortality and morbidity in patients with the appropriate anatomy of arteries and suitable conditions.

In most of the world's healthcare centers, patients with acute myocardial infarction are treated with agents, such as recombinant tissue plasminogen activator (rtPA), that facilitate restoration of blood flow. Treatment of acute ischemic stroke with rt-PA is effective in decreasing disability when it is prescribed in the first 3 hours after onset of ischemic stroke. Now, rtPA treatment is suggested as the only safe and effective treatment for cerebral ischemia ${ }^{2,10}$.

The present objective of this study was to determine the efficacy of treatment with rt-PA in improving the clinical status of acute ischemic stroke.

\section{METHODS}

\section{Patients}

This study was a clinical trial (code of ethics: IRCT201709049014N182) which was open to patients with acute ischemic stroke and whose medical records were referred to the Neurology Department of Farshchian Sina Hospital in Hamedan. The patients were admitted to the study from the beginning of April 2016 to the beginning of June Year 2017, and met the inclusion criteria.

The sample size in this study was as follows: 20 subjects in the treatment (experimental) group and 20 subjects in the control group. The control group was comprised of patients who were not eligible for treatment, based on candidate withdrawal criteria. Both groups were selected from patients who had been referred to the ER. To control confounding factors, the two groups were identical in terms of age, gender, and type of disease (all with stroke). The control group was selected from patients with acute ischemic stroke in the Neurology Department who did not undergo rt-PA injection.

\section{Treatment Agent}

The treatment group received tissue plasminogen activator (t-PA), a serine protease with corresponding gene in chromosome 8 (8P12) in humans. This protease catalyzes the transformation of plasminogen to plasmin, a major enzyme responsible for breaking down clots. Thus, it functions in decomposing clots containing fibrin, such as clots in cerebral thrombotic vascular lesions ${ }^{11}$.

Patients with suspected symptoms were visited by a primary care physician in the ER. In case of symptoms indicating acute ischemic stroke, neurology services were introduced and, if they had the criteria for entering the study, they were enrolled to the study.

\section{Ethical considerations}

In the process of collecting, recording and analyzing the final report, the confidentiality of patient information was maintained.

\section{Inclusion criteria}

The inclusion criteria included: age of 18 years and older, clinical diagnosis of acute ischemic stroke, measurable neuromuscular failure, and onset of symptoms at least 180 minutes before the onset of treatment.

\section{Exclusion criteria}

Based on the information obtained from the history of the disease and physical examination, computed tomography (CT) scans and evaluations of the brain were carried out. The study exclusion criteria were as follows: use of heparin within 48 hours and impaired partial thromboplastin time (PTT), use of warfarin and international normalized ratio (INR) $>1.7$, severe stroke within the past 3 months, gastrointestinal bleeding or thromboembolism during the past 3 weeks, major surgery in the past 2 weeks, arterial puncture at an inaccessible compressive site during the week before, previous intracerebral hemorrhage (ICH) at any time, brain tumor, arteriovenous malformation (AVM), cerebral aneurysm, seizures at the onset of symptoms, pregnancy, self-improvement of neurological symptoms, evidence of active bleeding during examination, presence of fractured, isolated or mildly defective nerves, non-debilitating clinical manifestations similar to subarachnoid hemorrhage (SAH), systolic hypertension above 185 or diastolic over 110 , glucose lower than 50 , platelets below 100,000, hyper-density indicating bleeding, hypodensity of more than one-third of brain hemisphere, and age over 80 years old.

\section{Experimental design and treatment}

Patient demographic data were completed by questionnaire. To evaluate the conditions for rt-PA injection, the patients were subjected to initial CT scan 
testing. The patients were then transferred to the intensive care unit (ICU). All injections were performed from the onset of the patient's symptoms until ICU arrival, within 4.5 hours to allow the patient to receive the injection. In the ICU, patients in the treatment group were infused with rt-PA $(0.9 \mathrm{mg} / \mathrm{kg}$ to $\max 90$ $\mathrm{mg} / \mathrm{kg}, 10 \%$ bolus) over a $60-\mathrm{min}$ period. The patients were then observed for signs of intracerebral hemorrhage. Determination of the response rate to the treatment at post-injection hours was conducted using the National Institutes of Health Stroke Scale (NIHSS). Another criterion for determining the outcome of the treatment group (after 90 days), compared to the control group, was the modified Rankin Scale (MRS).

\section{Study Limitations}

Some limitations of this study were that the start time of the stroke process was obscure, it was challenging to decide on when to start rt-PA treatment, and the examinations of neurological disorders were evaluated by different individuals in the study.

\section{Statistical analysis}

Data analysis was performed using SPSS software version 16. Quantitative data were shown (mean, standard deviation, frequency ratios, etc.), as well as qualitative data (tables, graphs, etc.).

\section{RESULTS}

The results of the study showed that all patients in the treated (case) group and the untreated (control) group had risk factors for ischemic stroke. The highest risk factor in the treatment and control groups was high blood pressure (55\%). The history of cardiovascular disease (CVD) in the treatment and control groups was estimated as $50 \%$. More than one risk factor was reported in about $50 \%$ of the treatment group and $65 \%$ of the control group.

CVD/Smoking was estimated as $5 \%$ in the treatment group and nil in the control group. Moreover, DM/Smoking was estimated as $15 \%$ in the control group as compared to nil in the treatment group (Table 1).

The results of the study showed that the mean age in the treatment group and control group were not significantly different. However, the mean of ER arrival time, NIHSS before treatment, and NIHSS on the 3rd day of treatment for the treatment group were higher than those for the control group; the differences were statistically significant $(\mathrm{P}<0.05)$ (Table 2$)$.

The results of the study showed that in the rt-PA treatment group, the difference between the mean
Pre-NIHSS-Post-NIHSS was 1.2 , which indicates a trend of decrease in neurological disability at day 3 of treatment compared to pre-treatment period; however, this difference was not statistically significant $(\mathrm{P}>0.05)$. In the control group, the difference for Pre-NIHSS-Post-NIHSS was -0.05 , which indicated that most of the neurological disabilities might occur on/after day 3 of treatment but this difference, too, was not statistically significant $(\mathrm{P}>0.05)$ (Table 3$)$. Our findings showed that probability of recovery of neurologic disabilities in the experimental group is higher than in the control group $(R R=1.25)$ and also odd ratio for receiving rt-PA in the group which had positive post-treatment NIHSS - pre-treatment NIHSS compared with the control group was larger than one. It indicates that the odds of getting treatment in the group with positive Pre-treatment NIHSS-Post-treatment NIHSS was more than the control group $(\mathrm{OR}=1.5)$.

Furthermore, the results showed that among the 20 patients in the treatment group, $6(30 \%)$ patients experienced complications of lung edema (15\%), high blood pressure (5\%), systemic hemorrhage (5\%), as well as an intracranial hemorrhage (Figure 1).

In general, the mortality rate was estimated as 13 cases in both groups, 7 cases in the treatment group, and 6 in the control group. There was no significant difference between the treatment and control groups in terms of mortality $(\mathrm{P}=0.5)$.

\section{DISCUSSION}

In recent years, improvements have been made in the treatment of acute ischemic stroke. One of the most important points to emphasize is that in the event of symptoms of ischemic stroke, the patient should immediately be sent to a facility equipped with the necessary facilities to start medical treatment. When the time interval between onset of symptoms and the onset of treatment is decreased, the less the effect of disability from the illness and, hence, a better prognosis. Trials designed by National Institute of Neurological Disorders and Stroke (NINDS) showed that acute ischemic stroke treatment using rt-PA is effective in reducing disability, if it is administered 3 hours later. The use of intravenous rt-PA, nowadays, is considered to be an essential component of primary stroke centers. In fact, it is the first treatment that has been proven to improve clinical outcomes in ischemic stroke. In reviewing the efficacy of rt-PA in 1995, NINDS showed that intravenous rt-PA was associated with a $30 \%(15 \%-11 \%)$ decrease in relative risk ${ }^{11}$. In the current study, we show that treatment with rtPA reduces the neurological disability in patients with 


\begin{tabular}{lccc}
\hline Table 1: Distribution of ischemic stroke risk factors in the treatment group and control group & \\
\hline \multicolumn{1}{c}{ Variable } & $\begin{array}{c}\text { Frequency (\%) } \\
\text { Treatment group } \\
(\mathbf{n = 2 0})\end{array}$ & Control group (n=20) & Total (n=40) \\
\hline High Blood pressure & $11(55)$ & $11(55)$ & $22(55)$ \\
CVD & $10(50)$ & $10(50)$ & $20(50)$ \\
DM & $6(30)$ & $7(35)$ & $13(32)$ \\
Smoking & $2(10)$ & $4(20)$ & $6(15)$ \\
More than 1 risk factor & $10(50)$ & $13(65)$ & $23(57)$ \\
CVD/DM & $2(10)$ & $3(15)$ & $5(12.5)$ \\
CVD/HTN & $3(15)$ & $4(20)$ & $7(12.5)$ \\
CVD/Smoking & $1(5)$ & $0(0)$ & $1(2.5)$ \\
HTN/DM & $3(15)$ & $0(0)$ & $3(7.5)$ \\
HTN/CVD/CVA & $1(5)$ & $2(10)$ & $3(7.5)$ \\
DM/Smoking & $0(0)$ & $3(15)$ & $3(7.5)$ \\
HPL/HTN & $0(0)$ & $1(5)$ & $1(2.5)$ \\
\hline
\end{tabular}

CVD: cardiovascular disease; CVA: cerebrovascular accident; DM: diabetes mellitus; HPL: hyperlipidemia; HTN: hypertension

Table 2: Relationship between the variables evaluated in the treatment group and the control group

\begin{tabular}{lccc}
\hline \multicolumn{1}{c}{ Variable } & \multicolumn{2}{c}{ Mean } & P-value \\
& Treatment group $(\mathbf{n = 2 0})$ & Control group $(\mathbf{n = 2 0})$ & 0.3 \\
\hline Age & 72.5 & 76.1 & 0.001 \\
Time to reach ER & 89.8 & 331.7 & 0.011 \\
NIHSS before treatment & 10.8 & 16.9 & 0.009 \\
$\begin{array}{l}\text { NIHSS on the 3rd day of } \\
\text { treatment }\end{array}$ & 9.6 & 9.6 & \\
\hline
\end{tabular}

ER: emergency room; NIHSS:National Institutes of Health Stroke Scale

Table 3: The difference between the mean Pre-NIHSS-Post-NIHSS in the treatment group and the control group

\begin{tabular}{lcccc}
\hline & Variable & Difference in Mean & \%95 CI & P-value \\
\hline $\begin{array}{l}\text { Pre-NIHSS- } \\
\text { Post-NIHSS }\end{array}$ & Treatment group & 1.2 & $(-0.2,2.6)$ & 0.09 \\
& Control group & -0.5 & $(-1.29,1.19)$ & 0.9
\end{tabular}

ischemic stroke. The mean of the onset of symptoms to time of arriving in the ER was significantly lower in the experimental (rt-PA treatment) group than in the control group.

The mean of NIHSS can reflect the severity of the neurologic disabilities resulting from ischemic stroke. In the treatment group, the NIHSS before treatment ("pre-treatment") versus on the 3rd day after treatment ("post-treatment") was lower than the NIHSS pre/post for the control group. The relative risk for NIHSS pre-treatment prevention was 1.25 , indicating that the rate of improvement in neurological disability in the rt-PA group was greater than that in the other group. Also, odd ratio for receiving rt-PA in the group which had positive post-treatment NIHSS - pre-treatment NIHSS compared with the control group was equal to 1.5. It indicates that the odds of getting treatment in the group with positive Pretreatment NIHSS-Post-treatment NIHSS was more than the control group.

Early recanalization after rt-PA injection greatly improves the outcome of patients. However, intra- 


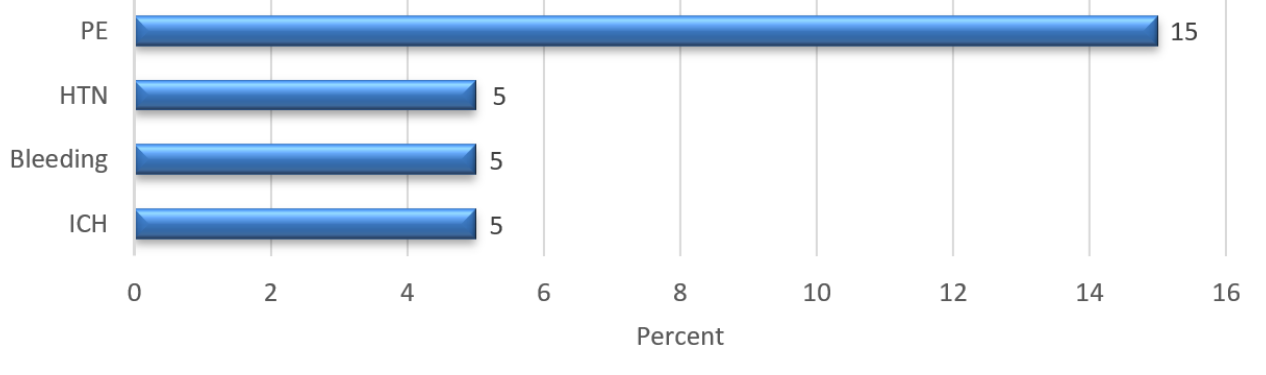

Figure 1: The prevalence of complications in the rt-PA treated group. PE: pulmonary edema; HTN: hypertension; ICH: intracranial hemorrhage.

venous thrombosis with rt-PA accounts for about $50 \%$ of stroke cases that successfully canalize the arteries. The onset of stroke until treatment is probably an important factor determining the next outcome ${ }^{1}$.

Some types of acute ischemic stroke treatments include venous thrombolytic therapy, arterial thrombolytic therapy, non-pharmacological therapy (mechanical embolectomy), surgery, and supportive and therapeutic interventions. Among the treatments reported, rt-PA injection has been considered as the most available therapy accessible at most neurological centers.

In one study, after excluding participants over the age of 80 , as well as those with severe stroke and diabetic cases, significant findings were obtained from tissue plasminogen injection. Thus, greater accessibility to visits by a neurologist could increase the number of injections in the eligible patients and improving the status of stroke patients. The causes of non-injection might be owing to the lack of correct diagnosis, the lack of imaging facilities, the lack of recognition of the patient's surroundings, the lack of recognition of primary care physician, and the incorrect transferring of the patient ${ }^{12}$.

Our study confirms the benefits of rt-PA treatment since treatment led to reduction of neurological disability in patients with ischemic stroke. Indeed, we showed that the mean of onset of symptoms (until arrival at the ER) was significantly lower in the treatment group than in the control group. Previous analysis by Marler et al. ${ }^{13}$ and Hacke et al. ${ }^{14}$ have shown that there is a direct relationship between time and effect of treatment.

In our study, the criteria for assessing the difference in NIHSS before and after treatment were the judgment criteria for the effectiveness of the drug, which was shown to be lower in the NIHSS-treated group than before treatment. In a study by Guettier et al. in 2016, neurological premature improvement at 1 hour and neurological improvement within the first 24 hours after treatment were defined by improving NIHSS by $40 \%$ of baseline. Of the 421 patients, $51 \%$ had a very early neurological recovery and $26 \%$ had a neurological early recovery ${ }^{12}$.

In a study by Li et al. (2013), primary neurological healing and continuous neurological healing in the first 24 hours were strong predictors of favorable outcome for 3 months in patients with acute ischemic stroke undergoing thrombolysis ${ }^{15}$. Also, in a 2010 study by Kazumi Kimura et al., the amount of premature recanalization from the onset of stroke to rt-PA injection in patients with acute ischemic stroke should begin as soon as possible ${ }^{1}$.

There are challenges in using tPA, which include the risk of marked intracerebral hemorrhage and lack of efficacy in some high-risk groups in the community. Recent American data suggest that the incidence of stroke will more than double by 2050 . Therefore, better training and coordination among physicians is necessary to optimize the use of rt-PA for all patients with stroke.

\section{CONCLUSION}

Treatment with rt-PA reduces the neurological disability in patients with ischemic stroke. The median MRS is lowered after 90 days in the treatment group, compared to the control group, thereby indicating a decrease in the long-term neurologic disorder in the treatment group. In light of the beneficial effects of rtPA treatment in this study, it is recommended that patients with acute stroke be treated with rt-PA as soon as possible. The benefits of early injection of this agent should be known to all neurologists, general practitioners and ER physicians, who are in the first line of dealing with such patients. 


\section{COMPETING INTERESTS}

The authors declare that there is no conflict of interest.

\section{AUTHORS' CONTRIBUTIONS}

All authors contributed to the design of the research. AGH, EG, and MKH collected the data. ZKH, EG conducted analysis and interpretation of data. All authors drafted the first version. MKH, ZKH, EG, and AGH edited the first draft. All authors reviewed, commented and approved the final draft.

\section{FINANCIAL SUPPORT}

This research is the result of a dissertation in the general population with a code identifier IRCT201709049014N182 from the Faculty of Medicine of Hamedan University of Medical Sciences, supported by Hamedan University of Medical Sciences.

\section{ABBREVIATIONS}

AVM: Arteriovenous Malformation

DM: Diabetes Mellitus

ICH: Intra-cerebral Hemorrhage

OCP: Oral Contraceptive Pills

RCT: Randomized Clinical Trial

SAH: Subarachnoid Hemorrhage

\section{REFERENCES}

1. Kimura K, Iguchi Y, Shibazaki K, Aoki J, Watanabe M, Matsumoto $\mathrm{N}$. Early stroke treatment with IV t-PA associated with early recanalization. Journal of the Neurological Sciences. 2010;295:53-7. Available from: DOI:10.1016/j.jns.2010.05.012.

2. Mouradian MS, Senthilselvan A, Jickling G, McCombe JA, Emery DJ, Dean N. Intravenous rt-PA for acute stroke: comparing its effectiveness in younger and older patients. Journal of Neurology, Neurosurgery, and Psychiatry. 2005;76:1234-7. Available from: DOI:10.1136/jnnp.2004.047803.

3. Poorthuis MH, Algra AM, Algra A, Kappelle LJ, Klijn CJ. Femaleand male-specific risk factors for stroke: a systematic review and meta-analysis. JAMA Neurology. 2017;74:75-81. Available from: DOI:10.1001/jamaneurol.2016.3482.
4. Jokela M, Pulkki-Råback L, Elovainio M, Kivimäki M. Personality traits as risk factors for stroke and coronary heart disease mortality: pooled analysis of three cohort studies. Journal of Behavioral Medicine. 2014;37:881-9. Available from: DOI:10.1007/s10865-013-9548-z.

5. Lightbody CE, Clegg A, Patel K, Lucas JC, Storey H, Hackett ML et al.. Systematic Review and Meta-Analysis of Psychosocial Risk Factors for Stroke; 2017.

6. Toni D, Angelantonio ED, Mascio MTD, Vinisko R, Bath PM, and PSG. Types of stroke recurrence in patients with ischemic stroke: a substudy from the PRoFESS trial. International Journal of Stroke. 2014;9:873-8. Available from: DOI:10.1111/ijs. 12150.

7. Fang J, Yuan K, Ayala C, Gindi R, Ward B. Abstract WP175: Stroke Prevalence Among US-and Foreign-born Adults in the United States; 2016

8. Krueger H, Koot J, Hall RE, O'Callaghan C, Bayley M, Corbett D. Prevalence of individuals experiencing the effects of stroke in Canada: trends and projections. Stroke. 2015;46:2226-31. Available from: Doi:10.1161/strokeaha.115.009616.

9. Adeloye D. An estimate of the incidence and prevalence of stroke in Africa: a systematic review and meta-analysis. PLoS One. 2014;9:e100724. Available from: DOI:10.1371/journal pone. 0100724 .

10. Fugate $J E$, Rabinstein $A A$. Absolute and relative contraindications to IV rt-PA for acute ischemic stroke. The Neurohospitalist. 2015;5:110-21. Available from: Doi:10.1177/ 1941874415578532.

11. Chapman SN, Mehndiratta $P$, Johansen MC, McMurry TL, Johnston KC, Southerland AM. Current perspectives on the use of intravenous recombinant tissue plasminogen activator (tPA) for treatment of acute ischemic stroke. Vascular Health and Risk Management. 2014;10:75-87.

12. Guettier S, Cogez J, Bonnet AL, Dean P, Apoil M, Tchoumi T. Factors associated with timing of early neurological improvement after thrombolysis for ischaemic stroke. European Journal of Neurology. 2016;23:664-7. Available from: DOI 10.1111/ene.12943.

13. Marler JR, Tilley BC, Lu M, Brott TG, Lyden PC, Grotta JC. Early stroke treatment associated with better outcome: the NINDS rt-PA stroke study. Neurology. 2000;55:1649-55. Available from: Doi:10.1212/wnl.55.11.1649.

14. Hacke W, Donnan G, Fieschi C, Kaste M, Broderick JP, Brott T, et al. Association of outcome with early stroke treatment pooled analysis of ATLANTIS, ECASS, and NINDS rt-PA stroke trials. 2004;

15. Yeo LL, Paliwal P, Teoh HL, Seet RC, Chan BP, Wakerley B. Early and continuous neurologic improvements after intravenous thrombolysis are strong predictors of favorable longterm outcomes in acute ischemic stroke. Journal of Stroke and Cerebrovascular Diseases. 2013;22:e590-6. Available from: DOI:10.1016/j.jstrokecerebrovasdis.2013.07.024. 